\title{
ACCRUALS DISCRICIONÁRIOS E PREVISÕES OTIMISTAS DOS ANALISTAS: INCENTIVOS E CONSEQUÊNCIAS
}

\author{
Paulo Victor Gomes Novaes 1 \\ Pedro Borges Júnior2 \\ José Elias Feres de Almeida3 \\ Patrícia Maria Bortolon4
}

- Artigo recebido em: 01/09/2016 -" Artigo aceito em: 02/04/2018 -" Segunda versão aceita em: 09/04/2018

\section{RESUMO}

O objetivo do estudo foi analisar a relação de incentivo existente entre previsões otimistas de analistas de mercado e o gerenciamento dos accruals discricionários. Foram utilizadas três variações do modelo de Jones Modificado. A amostra é composta por um conjunto de dados em painel desbalanceado, correspondente ao período de 2008 a 2013, composto de 80 empresas de capital aberto, com ações negociadas na BMF\&Bovespa e que tiveram todas as variáveis utilizadas no modelo, perfazendo o montante de 394 observações. Foram selecionadas empresas acompanhadas por pelo menos um analista de mercado em cada ano. As variáveis dos modelos foram coletas por meio do banco de dados do Comdinheiro, enquanto as informações de previsões e erros dos analistas foram obtidas por meio do sistema Thomson Reuters Eikon. Foi utilizado dados em painel com efeitos fixos para estimação dos modelos empíricos. Os resultados não sustentam as duas hipóteses desenvolvidas no estudo, quais sejam, a de que os accruals discricionários aumentam após erros de previsão otimistas, e que a manipulação de resultados influencia as previsões subsequentes dos analistas. Os resultados são robustos a diferentes estimadores e sub-amostra utilizados.

Palavras-chave: Gerenciamento de resultados; accruals discricionários; previsão de analistas.

\footnotetext{
1 Mestre em Contabilidade e Finanças pela Universidade Federal do Espírito Santo - UFES. Professor da Faculdade Multivix/Vitória. Endereço: R. José Alves, 135, Goiabeiras, Vitória-ES. Telefone: 27 3335-5666. CEP 29075- 080 - Vitória - Brasil - E-mail: pvgnovaes@hotmail.com.

2 Mestre em Contabilidade e Finanças pela Universidade Federal do Espírito Santo - UFES. Professor da Universidade Federal do Pará - UFPA. Endereço: R. Augusto Corrêa, 1 - Guamá, Belém - PA, 66075-110. Telefone: 91 99377-4178. E-mail: pedroborges@ufpa.br.

3 Doutor em Controladoria e Contabilidade pela Universidade de São Paulo - USP. Professor da Universidade Federal do Espírito Santo. Endereço: Av. Fernando Ferrari, 314, Centro de Ciências Jurídicas e Econômicas - Campus Goiabeiras. Telefone: 27 4009-2794. CEP: 29075-910 - Vitória/ES - Brasil - E-mail: jose.e.almeida@ufes.br.

4 Doutora em Finanças pela Universidade Federal do Rio de Janeiro - UFRJ. Professora da Universidade Federal do Espírito Santo. Endereço: Av. Fernando Ferrari, 314, Centro de Ciências Jurídicas e Econômicas - Campus Goiabeiras. Telefone: 27 4009-2794. CEP: 29075-910 - Vitória/ES - Brasil - E-mail: patricia.bortolon@ufes.br.
} 


\title{
DISCRETIONARY ACCRUALS AND MARKET ANALYSTS' OPTIMISTIC FORECASTS: INCENTIVES AND CONSEQUENCES
}

\begin{abstract}
This study aims to analyze the existing incentive' relation between optimistic market analyst forecasts and earnings management practices via discretionary accruals. We use three different approach of Jones Modified model. The sample consists of an unbalanced panel data, corresponding to the period from 2008 to 2013, composed by 80 public firms, with shares traded on BM\&FBovespa, totaling the amount of 394 observations. We select firms covered by at least one financial analyst for each year. The variables of the models were collected from Comdinheiro database, and the analyst forecasts and errors information were obtained from Thomson Reuters Eikon system. We use panel data with fixed effects to estimate the empirical models. The Results do not support the hypotheses developed in this study, as follow, that discretionary accruals increase after optimistic forecast errors, and the earnings management influences the subsequent analysts' forecasts. The results are robust for different estimators and subsample used.
\end{abstract}

Keywords: Earnings management. Discretionary accruals. Analysts forecast.

\section{INTRODUÇÃO}

Os analistas, enquanto intermediários informacionais, têm como principal função prever lucros e preços futuros de ações, de modo a recomendar os investimentos que tenderão a trazer os melhores resultados, de acordo com seus modelos. Para isso, se cercam de parâmetros e se comunicam com as companhias a fim de extrair informações técnicas úteis para a produção de seus relatórios e para emitir opiniões de compra e venda de ativos no mercado de capitais. Porém, nem as taxas de crescimento passadas das ações das empresas, tampouco o rendimento dessas ações são boas referências para prever as taxas futuras (CRAGG; MALKIEL, 1968).

De acordo com Abarbanell, Lehavy (2003), Degeorge, Patel e Zeckhauser, (1999), as previsões dos analistas têm impacto nas decisões de gerenciamentos de resultados das empresas. Todavia, Abarbanell e Lehavy (2003) sustentam que os analistas são incapazes ou desmotivados a prever completamente tais resultados. Sobretudo, os autores encontram evidências de associação entre grandes gerenciamentos de resultados para baixo (large-negative) e erros de previsões (previsões pessimistas), ao passo que a relação de incentivo de maior gerenciamento de resultado para cima também ocorre em favor das recomendações dos analistas.

Numa análise das surpresas das previsões dos analistas, definida como a diferença entre a última revisão dos analistas e o resultado divulgado pelas empresas, Martinez (2004) descobriu que os investidores brasileiros reagem às surpresas, sendo que penalizam as empresas antecipadamente quando já esperam más notícias. Anos mais tarde, o mesmo autor investigou se as empresas brasileiras gerenciam seus resultados para atender às demandas do mercado de 
capitais utilizando as métricas para capturar accruals discricionários (MARTINEZ, 2009).

Empresas com lucros antes da manipulação dos accruals estão mais propensas a usar os accruals para atingir as projeções dos analistas do que empresas com prejuízos antes da manipulação dos accruals (BARUA; JOSEPH LEGORIA; MOFFITTI, 2006). Por exemplo, Athanasakou, Strong e Walker (2009) encontraram evidências de que, no Reino Unido, as empresas estão mais inclinadas a direcionar as previsões do que gerenciar os accruals. Já Comprix, Muller e Stanford (2003) evidenciaram que empresas gerenciam resultados via despesas operacionais com o intuito de atingir ou superar as expectativas dos analistas.

As empresas que buscam somente alcançar as projeções dos analistas ao invés de superá-las, o fazem por não terem mais reservas de accruals, ou ainda porque os custos para gerenciar os resultados até o consenso já lhes são demasiadamente altos e excedem os benefícios de superar as expectativas dos analistas (LEE, 2007).

Almeida, Lopes e Corrar (2011) encontraram evidências de que as empresas brasileiras de capital aberto com índice Market-to-book superior a 1 são mais incentivadas a gerenciar os resultados. Martinez (2009), por sua vez, encontrou relação negativa entre o número de analistas e a magnitude dos accruals discricionários e os erros de previsão, o que sugere que maior monitoramento dos analistas inibe a prática de gerenciamento de resultados e, por consequência, aumenta a precisão do consenso dos analistas.

Keung, Lin e Shih (2010) defendem que os analistas reagem mal às empresas que alcançam ou minimamente superam suas expectativas, pois percebem isso como um sinal de manipulação de resultados. Os autores verificam que a relação entre as surpresas futuras e as atuais é negativa somente para empresas que atingem ou superam muito pouco os resultados esperados; os analistas preveem a ocorrência de gerenciamento de resultados e se utilizam disso para ajustar suas previsões futuras e ficam motivados a fazer previsões otimistas quando o gerenciamento é para maior. Porém, conforme Givoly, Hayn e Yoder (2011), os desempenhos posteriores das empresas não sustentam o otimismo.

Em estudo recente, Martins, Paulo e Monte (2016) apresentam evidências de que a relação entre o gerenciamento de resultados via accruals discricionários e o erro médio de previsão é sensível à proximidade dos resultados da empresa ao consenso dos analistas. Os gestores, portanto, sentem-se mais incentivados a gerenciar quando estão próximos dos valores projetados pelos analistas de mercado.

Mota et al. (2017), por sua vez, ratificam os resultados do mercado norteamericano apresentado por Comprix e Stanford (2003), ao encontrar evidências de que o uso de accruals discricionários pelos gestores tem o intuito de alcançar ou bater as previsões dos analistas. $O$ estudo nacional rejeita a hipótese de que empresas que apresentaram resultados acima do consenso dos analistas (ou seja, surpresa positiva) tenham sido incentivadas a gerenciar mais resultados, entretanto, para empresas com pequenas surpresas (valores do primeiro quartil) 
encontraram evidências de gerenciamento de resultados tanto para cima quanto para baixo.

O papel de monitoramento utilizando a informação contábil é tratado desde Jensen e Meckling (1976), que expõem que as diversas forças do mercado, como lobby, covenants e expectativa de valorização do preço da ação e pagamento de dividendos, incentivam o atingimento de tais metas. Nesse cenário, os analistas acabam por atuar como monitores dos acionistas, em especial, dos minoritários. Consequentemente, as previsões se tornam benchmark para os investidores, e tais previsões se tornam alvo dos gestores (BEAVER, 2002), fechando o ciclo em uma tentativa de alinhamento de incentivos, mas com bases informacionais potencialmente manipuladas (BARTOV; GUL; TSUI, 2001).

Os diferentes achados aumentam a motivação para questionamentos sobre a origem do incentivo ao gerenciamento dos resultados, bem como aos benefícios trazidos por tal prática. Desta forma, este estudo se propõe a responder a seguinte questão: Qual é a relação entre previsões otimistas dos analistas de mercado em um período e o gerenciamento do resultado na direção do atingimento da expectativa do mercado para ano seguinte?

Assim, o objetivo do estudo é analisar a relação entre previsões otimistas de analistas de mercado e gerenciamento de resultados por meio dos accruals discricionários. Previsões otimistas são também chamadas de surpresas negativas, pois só é possível classificar uma previsão como otimista depois que a empresa divulga seu resultado (lucro por ação) e, então, se constata se a surpresa foi positiva (previsão pessimista) ou negativa (previsão otimista).

Não foram encontradas evidências de que os accruals discricionários influenciam o otimismo dos analistas ou que as empresas aumentam os accruals discricionários por incentivo advindo do mercado via previsão dos analistas. Os resultados são robustos a diferentes estimadores utilizados (não reportados) e uma sub-amostra contendo somente empresas com previsões otimistas. Os resultados reforçam evidência anterior de Mota et al. (2017).

O artigo está organizado nesta sequência: introdução, referencial teórico e desenvolvimento das hipóteses, metodologia, discussão dos resultados e considerações finais.

\section{REFERENCIAL TEÓRICO E DESENVOLVIMENTO DAS HIPÓTESES}

\subsection{Contabilidade e Analistas de Mercado}

A abordagem positiva da contabilidade comprovou que a ciência passou a assumir um posto de fornecedora de informações que auxiliava não só as decisões internas, mas também dava suporte para projeções de lucro e previsões de preço de ações. Com isso, surgiu uma expectativa ao redor da estreita relação entre a contabilidade e os analistas de mercado, isto é, profissionais que aplicam seus conhecimentos técnicos no intuito de realizar recomendações de compra, manutenção e venda, bem como fazer previsões sobre o resultado de uma empresa em um período determinado (CRAGG; MALKIEL, 1968).

As empresas, por outro lado, não esperam até o final do ano para alcançar ou superar as expectativas dos analistas, esse jogo é contínuo, de modo que os retornos anormais por trimestre estão positivamente associados com as surpresas 
positivas em relação ao erro de previsão dos analistas (BARTOV; GUL; TSUI, 2001). Não obstante, prever quanto uma empresa auferirá de lucro não é uma tarefa fácil, considerando a complexidade do mercado e a diversidade de ações que as empresas tomam diariamente visando manterem-se vivas e atuantes no mundo dos negócios (MARTINEZ, 2001).

\title{
2.2. Accruals Discricionários
}

A prática de gerenciamento de resultados por meio de accruals discricionários tem sido investigada sob diversas abordagens, buscando informações sobre os incentivos do gerenciamento e formas de identificação, melhorando a capacidade de análise tanto do investidor quanto dos órgãos reguladores de mercado (ALMEIDA; DALMÁCIO, 2015; ATHANASAKOU; STRONG; WALKER, 2009; BARUA; LEGORIA; MOFFITTI, 2006; DALMÁCIO et al., 2013; MARTINEZ, 2009).

Barua et al. (2006) estudaram se empresas que apresentam lucros antes de manipular os accruals estão mais inclinadas a gerenciar resultados para atingir as expectativas dos analistas do que empresas que possuem prejuízo antes da manipulação dos accruals. Os autores evidenciaram que as primeiras estão mais propensas a ter resultados gerenciados tanto para atingir as projeções dos analistas quanto para atingir os benchmarks representados pelos resultados de períodos anteriores.

As empresas se utilizam de outras formas de gerenciamento de resultados, conforme Athanasakou et al. (2007, p.14):

\begin{abstract}
As both managers and analysts tend to exclude non-recurring items from core earnings, classification shifiting of core expenses or losses to non-recurring items after the end of the accounting period and before the announcement of the results could increase the probability of hitting the final forecast.
\end{abstract}

Desse modo, elas direcionam as previsões dos analistas e, por conseguinte, atingem as expectativas dos analistas. No Reino Unido, as empresas não se utilizam de accruals de capital de giro anormais para atender às expectativas dos analistas (ATHANASAKOU; STRONG; WALKER, 2009).

Como algumas companhias recebem atenção maior de analistas do que outras-mensurado pelo número de analistas que acompanham a firma-, espera-se que a precisão na previsão dos analistas aumente à medida que o número de olhos sobre a empresa cresça. Ainda, sobre o número de analistas que acompanha uma empresa, também se reconhece a função de monitores que os analistas exercem sobre elas, limitando as tentativas de gerenciamento dos resultados (MARTINEZ, 2009).

Analisando o período de 2000 a 2008, Dalmácio et al. (2013) encontraram evidências de que empresas com maior nível de governança corporativa sinalizavam maior qualidade informacional para o mercado, resultando em maior acurácia dos analistas. Contudo, Erfurth e Bezerra (2013) rejeitaram a hipótese de diferenças no nível de gerenciamento de resultado via accruals discricionários, de 2000 até 2007, evidenciando que o engajamento em práticas diferenciadas 
de governança corporativa não interferiu nesse tipo de comportamento dos gestores.

Resultado semelhante foi encontrado anos mais tarde, por Silva (2015), que buscava identificar o efeito da adoção das IFRS no gerenciamento de resultado, estudando as empresas não-financeiras no período de 2003 a 2014. Para garantir a robustez do resultado, o autor aplicou os diversos modelos de gerenciamento de resultado (por accruals e operacionais), mas a única sinalização é de que as empresas com maior nível de gerenciamento diminuíram essa prática após a adoção das normas internacionais.

Ainda, Almeida, Lopes e Corrar (2011) identificaram que empresas com maiores oportunidades de crescimento, observada pela relação entre o preço de mercado e o valor contábil da ação (Market-to-Book - MTB), tendem a gerenciar resultados. Os resultados sinalizaram diferença significativa no nível de gerenciamento de resultado via accruals discricionários entre empresas com MTB acima de 1 em relação às outras. Desta forma, os gestores parecem gerenciar a expectativa dos agentes econômicos pela geração de caixa futuro da empresa.

Aspectos relacionados à estrutura de propriedade parecem ter a capacidade de inibir a prática de gerenciamento de resultados. Holanda e Coelho (2012) demonstram de forma mais consistente que a existência de acionistas preferencialistas, por exemplo, está significativamente relacionada à redução do gerenciamento. Contudo, deve-se considerar que o estudo apresenta resultados conflitantes para os diferentes tipos de proxies de gerenciamento de resultado.

Lima et al. (2015), em outra abordagem, não identificaram distinção na prática do gerenciamento via accruals entre os estágios do ciclo de vida das empresas brasileiras, muito embora a teoria seja utilizada para explicar os resultados que apontaram maior acurácia dos analistas em empresas maduras, ao passo que empresas classificadas como em turbulência presenciaram maior dispersão dos analistas de mercado (OLIVEIRA; GIRÃO, 2016).

A expectativa dos analistas constitui um incentivo para o gerenciamento de resultados, que pode ser feito por meio dos accruals discricionários que a companhia tem disponível. Se a expectativa dos analistas ficou além do que o resultado evidenciado pela empresa, espera-se que a empresa se utilize dos accruals para evidenciar um resultado que se aproxime daquele previsto pelos analistas. Nesse contexto, este estudo apresenta a primeira hipótese:

\section{$H_{1}$ : A utilização dos accruals discricionários aumenta, dado que no ano anterior o resultado da empresa ficou abaixo da expectativa dos analistas.}

\subsection{Gerenciamento de Resultados e Previsões de Analistas}

Na linha de pesquisa referente às contas de resultado que são mais propensas a serem utilizadas para gerenciamento, Plummer e Mest (2001) evidenciam que as receitas de vendas e as despesas operacionais são as mais usadas no gerenciamento. Dessa forma, empresas recomendadas pelos analistas para compra gerenciam os resultados para cima, ao contrário das empresas recomendadas para venda. 
Matsumoto (2002) estudou quais seriam os incentivos para os gestores evitarem as surpresas negativas, pois embora a literatura explore exaustivamente - gerenciamento de resultados como ferramenta para atender as metas de resultados, pouco se estuda sobre o direcionamento das previsões dos analistas. Especificamente, se os analistas não antecipam completamente, em suas previsões, os efeitos de gerenciamento de resultados das empresas, haverá associação entre o gerenciamento de resultados daquela empresa e o sinal e a magnitude dos erros de previsão dos analisas.

É esperado que o erro dos analistas aumente ou diminua em função da sensibilidade ex-ante do preço das ações às notícias de resultados. Existem variáveis de mercado ex-ante (modelo de Jones modificado) que conseguem prever tanto o sinal quanto a magnitude do gerenciamento de resultados da empresa e do erro de previsão dos analistas (ABARBANELL; LEHAVY, 2003).

Os analistas fazem uma previsão no início do ano e depois fazem correções, ajustes ou reforçam suas previsões ao longo do ano até a data da publicação do resultado pela empresa analisada. A surpresa ocorre quando a empresa divulga um resultado diferente daquele previsto pelos analistas (e, consequentemente, esperado pelo mercado). Surpresas positivas-divulgações que superam as expectativas - são premiadas com aumento do valor da ação; já as surpresas negativas, aquelas divulgações de resultado que são inferiores ao esperado, provocam decréscimo no valor da ação. Essa diferença constitui o erro de previsão dos analistas. Portanto, o lucro contábil é relevante para os investidores e o mercado premia as empresas que superam positivamente as previsões dos analistas (MARTINEZ, 2004).

Além disso, os gestores das empresas têm interesse em aumentar sua remuneração e o valor de suas opções de ações. Porém, eles podem adotar a estratégia de gerenciar os resultados para baixo e manipular as previsões dos analistas e, no momento oportuno, apresentar um resultado que supera as expectativas dos analistas "gerenciadas" para baixo. Por fim, as empresas que constantemente superam as previsões são reconhecidas pelo mercado e tem suas ações valorizadas (LEE, 2007).

As empresas tendem a gerenciar seus resultados para atender a três objetivos: diminuir a possibilidade de divulgar perdas, manter o desempenho atual ou, se for o caso, tornar o resultado o pior possível (big bath) para não prejudicar os resultados posteriores (MARTINEZ, 2009).

Quanto maior a experiência do analista, pior é sua acurácia na previsão (exceto para empresas acompanhadas há vários anos), que não tem relação significativa com o tamanho da carteira de empresas e a diversificação de setores cobertos. Mesmo assim, as evidências mostram que os analistas das corretoras brasileiras são tão precisos quanto os das corretoras estrangeiras, e o desempenho anterior explica consideravelmente o desempenho futuro do analista (MARTINEZ, 2009).

Almeida e Dalmácio (2015) acrescentam evidências de que ambientes mais competitivos estimulam um fluxo maior de informações, que não necessariamente se traduz em maior qualidade. Sobretudo, os autores 
evidenciam que existe relação entre maior nível de governança e a acurácia das previsões dos analistas de mercado.

Se a previsão dos analistas funcionar como um termômetro para o caminho que os resultados evidenciados pela companhia devem seguir, seja para além ou aquém do resultado real, espera-se que o gerenciamento de resultados ocorra indo ao encontro daquele valor previsto pelos analistas, com a intenção de diminuir o efeito surpresa. Conforme a discussão proposta e como forma de verificar a hipótese 1, a segunda hipótese do trabalho visa testar se:

\section{$\mathrm{H}_{2}$ : A manipulação dos resultados em um período influencia as previsões dos analistas no período subsequente.}

\section{METODOLOGIA}

O estudo se propõe a analisar a influência das previsões otimistas na prática de gerenciamento de resultado, aproximada pela utilização dos accruals discricionários. Para isso, foram adotadas três versões do modelo Jones, modificado por Dechow et al. (1995), Kothari, Leone e Wasley (2005) e Dechow, et al. (2012).

\subsection{Seleção Da Amostra}

A amostra utilizada no estudo consiste em um conjunto de dados em painel, correspondente ao período de 2008 a 2013, composto de 80 empresas de capital aberto, com ações negociadas na Bovespa e que tiveram disponíveis todas as variáveis utilizadas nos modelos, mas não necessariamente para todos os anos, perfazendo o montante de 394 observações, o que configura um painel desbalanceado.

Cabe ressaltar que amostra ficou também restrita às empresas que foram seguidas por pelo menos um analista de mercado. As variáveis foram coletas por meio do banco de dados Comdinheiro, enquanto as informações de previsões e erros dos analistas foram obtidas por meio do sistema Thomson Reuters Eikon.

\subsection{Variável Dependente}

O modelo Jones Modificado utiliza os resíduos do modelo de regressão dos Accruals Totais e tem como proposta captar os accruals anormais, que representam as escolhas dos gestores no intuito de deslocar o resultado para atingir determinados interesses (ALMEIDA; BRANDRÃO, 2009; DECHOW et al., 1995; RONEN; YAARI, 2008). E a construção da variável acontece da seguinte forma:

$$
A C T_{i, t}=\left(\Delta C A_{i, t}-\operatorname{Cash}_{i, t}-\Delta C L_{i, t}-\Delta S D_{i, t}-D E P_{i, t}\right) / A T_{t-1}
$$

Em que: $\mathbf{A C T}_{\mathbf{i}, \mathrm{t}}=$ Accruals totais da firma i no período t; $\Delta \mathbf{C A}_{\mathbf{i}, \mathrm{t}}=$ Variação no ativo circulante da firma i no período t; $\Delta$ Cash $_{i, t}=$ Variação em caixa $e$ 
equivalentes de caixa da firma i no período $t ; \Delta \mathbf{C L}_{\mathrm{i}, \mathrm{t}}=$ Variação em passivo circulante da firma i no período t; $\Delta$ STD $_{\mathrm{i}, \mathrm{t}}=$ Variação em financiamento de curto prazo no passivo circulante da firma i no período t; $\mathbf{D E P}_{\mathbf{i}, t}=$ Depreciação $e$ amortização da firma i no período $t ; \mathbf{A T}_{t-1}=$ Ativo total em t- 1 da firma i no período anterior. Alternativamente, foram utilizados o cálculo dos accruals totais indiretamente pela fórmula: $A C T=(L L-F C O) / A T_{t-1}$, em que $L L$ é o lucro líquido e FCO é o fluxo de caixa das operações e ACT = (LL+DEP\&AMORT-FCO)/ATt-1, em que DEP\&AMORT é o montante de depreciação e amortização no período (DECHOW; DICHEV, 2002; GIVOLY; HAYN, 2000).

Foram utilizadas três versões do modelo Jones, por meio dos quais o estudo pretende capturar a utilização da discricionariedade dos gestores. $O$ primeiro modelo é o modelo Jones Modificado (DECHOW et al., 1995):

$$
\text { ACTi, } t=a_{0}+a_{1}(1 / \text { ATt- } 1)+a_{2}(\triangle R E C-\Delta C R E C) / \text { ATt- } 1+a_{3}(\text { PPE/ATt-1) }+\varepsilon i, t
$$

Em que: $\mathbf{A} \mathbf{C T}_{\mathbf{i}, \mathrm{t}}=$ Accruals totais da firma i no período t; $\Delta \mathbf{R E C}_{\mathbf{i}, \mathrm{t}}=\mathrm{V}$ ariação da receita da firma i no período t; $\Delta \mathbf{C R E C}_{\mathbf{i}, t}=$ Variação de contas a receber da firma i no período t; $\mathbf{P P E}_{\mathrm{i}, \mathrm{t}}=$ Imobilizado da firma i no período t; e ATt-1 = Ativo total da empresa i no período anterior.

O modelo aprimorado por Kothari et al. (2005) captura efeitos da performance das empresas adicionando o retorno sobre o ativo total (ROA) ao modelo:

$$
\begin{aligned}
& \text { ACTi, } t=a_{0}+a_{1}(1 / \text { ATt- } 1)+a_{2}(\triangle R E C-\triangle C R E C) / \text { ATt- } 1+a_{3}(\text { PPE } / \text { ATt- } 1)+ \\
& a_{3} \text { ROA }+\varepsilon i, t
\end{aligned}
$$

Por fim, o terceiro modelo é uma versão mais recente aprimorada por Dechow et al. (2012), em que consideram a reversão dos accruals totais de um período para o outro:

$$
\begin{aligned}
& \text { ACTi,t= } a_{0}+a_{1}\left(1 / \text { ATt-1) }+a_{2}(\triangle R E C-\triangle C R E C) / \text { ATt- } 1+a_{3}(\text { PPE } / A T t-1)+\right. \\
& a_{3} A C T_{i, t-1}+\varepsilon i, t
\end{aligned}
$$

\subsection{Variáveis Explicativas}

Baseados inicialmente na proposta levantada por (Beaver, Lambert e Dale, 1980), que testou a influência do preço da ação nos lucros contábeis, contrariando o viés dos estudos da época, este modelo testará a influência do não atingimento do consenso dos analistas de mercado na utilização dos accruals discricionários. Com isso, testar-se-ão as variáveis:

Quadro 1: Variáveis explicativas de interesse 


\begin{tabular}{|c|c|c|}
\hline VARIÁVEL & SINTAXE & AUTORES \\
\hline ERROit-1 & ErroPrev $=\frac{L P A_{\text {real }}-L P A_{\text {prev }}}{/ L P A_{\text {real }} /}$ & $\begin{array}{c}\text { (DALMÁCIO et al., 2013; } \\
\text { MARTINEZ, 2004; SAITO; } \\
\text { VILLALOBOS, 2008) }\end{array}$ \\
\hline DOTIMit-1 & $\begin{array}{c}\text { Variável Dummy de Otimismo (1 para } \\
\text { previsão acima do realizado; e 0, caso } \\
\text { contrário) }\end{array}$ & (DALMÁCIO et al., 2013) \\
\hline DOTIM*ERRO & $\begin{array}{c}\text { Interação da variável dummy de otimismo } \\
\text { de previsão com a magnitude do erro. }\end{array}$ & (MARTINEZ, 2007) \\
\hline
\end{tabular}

Fonte: Elaborado pelos autores.

A variável $E R R O_{i t-1}$ não será incluída diretamente no modelo pelo fato de que a variável de interesse é oriunda majoritariamente dela. Desta forma, a inclusão das duas variáveis juntas aumentaria as chances de ferir a premissa de ausência de colinearidade perfeita entre os regressores.

\subsection{Variáveis de Controle}

As variáveis de controle têm um papel mais do que exploratório em uma função de regressão. Segundo Fávero et al. (2009), elas servem para isolar o efeito dos coeficientes das variáveis de interesse sobre a variável dependente (accruals discricionários). Desta forma, serão testadas as seguintes variáveis de controle:

- QANALIST - Representa a quantidade de analistas que acompanham a empresa i no tempo t. Espera-se uma relação negativa entre cobertura da empresa e gerenciamento de resultados (MARTINEZ, 2009; SAITO et al., 2008);

- DESVP_ERRO - Quanto os analistas divergem entre si sobre a previsão de uma empresa. Espera-se que a maior volatilidade das previsões tenha impacto positivo no gerenciamento de resultados, dada a relação de mesmo sinal para acurácia das previsões (SAITO; BENETTI; VILLALOBOS, 2008);

- ENDIVIDAMENTO - Representa a participação de capital de terceiros no financiamento de seus ativos (Passivo Total/Ativo Total). Espera-se observar uma relação negativa, tendo em vista a maior complexidade nos contratos, envolvendo taxas de juros, fornecedores e credores por empréstimo (BASU; HWANG; JAN, 1998). Com isso, menor será influência dos números contábeis nas previsões e, naturalmente, menor também será a resposta em manipulação dos accruals do período seguinte.

- TAMANHO - Representado pelo logaritmo do Ativo Total da empresa i no tempo $t$, espera-se que empresas maiores manipulem menos o seu resultado, considerando a maior complexidade dos contratos e maior necessidade de transparência junto às partes relacionadas (DALMÁCIO et al., 2013). 


\subsection{Modelos}

No intuito de analisar a influência do não atingimento do consenso dos analistas (no ano anterior) sobre a utilização de accruals discricionários, manipulando o resultado no sentido das previsões, foi utilizado o modelo de regressão com dados em painel. $O$ primeiro modelo utiliza os termos de erro da regressão dos accruals totais, confrontando-se com as variáveis explicativas citadas, e pode ser expresso pela equação 1 abaixo:

$$
A D_{i t}=\mathbf{Y}_{0}+\mathbf{Y}_{1} \text { DOTIM }_{\mathrm{it}-1}+\mathbf{Y}_{\mathbf{2}} \text { ERROPREV }_{\mathrm{it}-1}+\mathbf{Y}_{\mathbf{3}} \text { DOTIM }_{\mathrm{it-1}} * \text { ERROPREV }_{\mathrm{it}-1}+\sum_{\mathrm{j}}^{\mathrm{k}} \mathbf{Y}_{\mathbf{j}} \mathrm{VC}_{\mathrm{it}-1}^{\mathrm{j}}+\varepsilon_{\mathrm{it}}
$$

Em que: $\boldsymbol{A D}_{\text {it }}=$ Accruals Discricionários da empresa i no ano t; $\mathbf{D O T I M}_{\text {it-1 }}=$ Variável dummy que captura a previsão consensual otimista para $\bigcirc$ ano anterior; DOTIM $_{\text {it-1 }}{ }^{*}$ ERROPREV $_{\text {it-1 }}=$ Interação da variável dummy de otimismo com a magnitude do erro de previsão na empresa i no ano t-1; e $\sum_{j}^{k} \boldsymbol{Y}_{j} V C_{i t-1}^{j}=$ Representa o conjunto de variáveis de controle levantadas pela literatura, que objetivam o isolamento do efeito das variáveis de interesse sobre a variável dependente.

Com isso, espera-se que os coeficientes $\boldsymbol{Y}_{1}$ e $\boldsymbol{Y}_{3}$ sejam positivos $\mathrm{e}$ significativos, atendendo a hipótese 1, indicando que a utilização dos accruals discricionários aumenta, dado que no ano anterior o resultado da empresa ficou abaixo da expectativa dos analistas.

No intuito de atender a hipótese 2 deste estudo, isto é, que o gerenciamento dos resultados influencia as previsões, o modelo foi invertido para capturar a influência da utilização dos accruals discricionários na magnitude do erro, conforme a equação 2, abaixo:

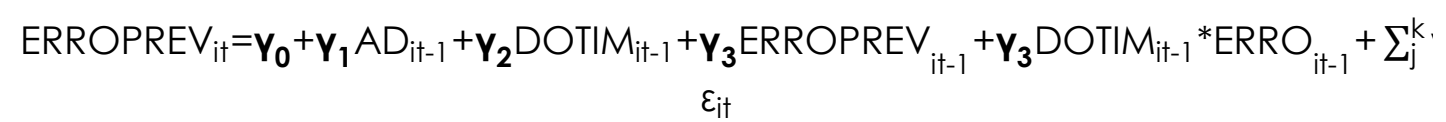

Em que: ERROPREV $\boldsymbol{~}_{\text {it }}$ é a variável dependente que representa a magnitude do erro no ano corrente. Desta forma, a variável explicativa $A D_{\text {it- } 1}$ (Accruals Discricionários no período anterior) buscará explicar se o erro diminuirá com o aumento do gerenciamento de resultados $\left(\mathrm{H}_{2}\right)$. Incluem-se também na relação das variáveis explicativas o erro no ano anterior e as outras variáveis já comentadas acima para captar alguma tendência errática ou tentativa de recuperar o erro de previsão do ano anterior (MARTINEZ, 2007). Portanto, esperase que o coeficiente $\boldsymbol{Y}_{1}$ seja negativo e significativo. 


\section{DISCUSSÃO DOS RESULTADOS}

\subsection{Estatística Descritiva e Correlação de Pearson}

Com base nos procedimentos descritos na metodologia e a partir dos dados coletados de suas respectivas fontes, a tabela 1 apresenta as estatísticas descritivas das variáveis utilizadas nos modelos.

Tabela 1 - Estatística Descritiva

\begin{tabular}{lcccccc}
\hline \multicolumn{1}{c}{ Estatísticas } & Num. Obs. & Média & Desv.Pad & p.25 & p.50 & p75 \\
\hline AD:JM & 394 & -0.02 & 0.15 & -0.09 & 0.00 & 0.05 \\
AD:KT & 394 & -0.02 & 0.15 & -0.09 & 0.00 & 0.05 \\
AD:DC & 394 & -0.02 & 0.15 & -0.08 & 0.00 & 0.05 \\
\hline Erroprev_t & 394 & -5.20 & 15.17 & -4.35 & -1.50 & -0.34 \\
Qanali_t & 394 & 11.70 & 5.35 & 7.00 & 13.00 & 16.00 \\
Dotimer_† & 394 & -0.01 & 0.07 & 0.00 & 0.01 & 0.02 \\
Desvio_† & 394 & 0.79 & 0.26 & 0.65 & 0.79 & 0.93 \\
Endiv_† & 394 & 2.21 & 2.97 & 0.86 & 1.41 & 2.23 \\
MTB_† & 394 & 2.66 & 5.13 & 0.92 & 1.50 & 2.62 \\
At_† & 394 & 22.68 & 1.48 & 21.54 & 22.61 & 23.65
\end{tabular}

Notas: AD: JM, AD:KT e AD:DC são Accruals Discricionários estimados pelos modelos propostos por Dechow et al. (1995), Kothari et al. (2005) e Dechow et al. (2012). Os demais ADs estimados foram suprimidos para facilitar a visualização da tabela, mas estão disponíveis, caso sejam solicitados; Qanali_t é o número de analistas que acompanharam a firma i no período t; Erroprev_t representa o erro da previsão da média dos analistas da firma i no período t; Dotimer_t é a interação de uma dummy que assume o valor 1 quando o erro foi menor do que zero (previsão otimista) com a magnitude do erro das previsões da firma i no período t; Desvio_t diz respeito à variabilidade das previsões da firma i no período t; Endiv_t apresenta o endividamento da empresa da firma i no período $t$, medido pela razão entre as dívidas de curto e longo prazo e o ativo total; At_t é logaritmo natural do ativo total da firma i no período t.

Todas as variáveis foram winsorizadas a $1 \%$ em cada cauda. Com base na tabela 1, é possível observar que todos os quartis da variável ERROPREV_† são negativos sugerindo que em todos os estratos da amostra os analistas, em geral, são otimistas. A média de analistas que acompanham as empresas é de 11,7, variando de 2 analistas no mínimo (Companhia Guararapes) e máximo de 24 (Companhia Energética de São Paulo - CESP), sendo que p.25 é de 7, a mediana 13 e p.75 de 16.

Sobre a variável dummy DOTIM, cabe ressaltar que ela apresentou uma média de 0,8223 , indicando que $82,23 \%$ das previsões do ano anterior foram otimistas, isto é, a maioria das empresas não atingiu a expectativa do mercado para os anos da amostra. 
A Tabela 2 apresenta os coeficientes da correlação de Pearson, acompanhados da significância estatística, no intuito de observar a força da relação, em uma extensão que varia entre -1 e 1, entre as variáveis estudadas.

Tabela 2 Correlação de Pearson

\begin{tabular}{|c|c|c|c|c|c|c|c|c|}
\hline Variáveis & AD:JM & AD:KT & AD:DC & Erroprev_t & At_t & Qanali_t & Desvio_t & Endiv_t \\
\hline \multirow{2}{*}{ AD:KT } & 0.9999 & 1 & & & & & & \\
\hline & 0.0000 & & & & & & & \\
\hline \multirow{2}{*}{$A D: D C$} & 0.9628 & 0.9627 & 1 & & & & & \\
\hline & 0.0000 & 0.0000 & & & & & & \\
\hline \multirow{2}{*}{ Erroprev_† } & 0.1067 & 0.1038 & 0.1209 & 1 & & & & \\
\hline & 0.0343 & 0.0395 & 0.0163 & & & & & \\
\hline \multirow{2}{*}{$A t \_\dagger$} & 0.0938 & 0.0967 & 0.1052 & 0.0638 & 1 & & & \\
\hline & 0.0629 & 0.0552 & 0.0369 & 0.2065 & & & & \\
\hline \multirow{2}{*}{ Qanali_† } & 0.0492 & 0.0501 & 0.0494 & 0.1206 & 0.4397 & 1 & & \\
\hline & 0.3301 & 0.3210 & 0.3278 & 0.0166 & 0.0000 & & & \\
\hline \multirow{2}{*}{ Desvio_† } & -0.0823 & -0.0807 & -0.0830 & -0.0455 & 0.1162 & 0.1298 & 1 & \\
\hline & 0.1028 & 0.1097 & 0.0998 & 0.3681 & 0.0211 & 0.0099 & & \\
\hline \multirow{2}{*}{ Endiv_† } & -0.1001 & -0.0960 & -0.0940 & 0.0166 & 0.1309 & 0.0259 & -0.0120 & 1 \\
\hline & 0.0471 & 0.0570 & 0.0622 & 0.7421 & 0.0093 & 0.6088 & 0.8128 & \\
\hline \multirow{2}{*}{ MTB_ $\dagger$} & -0.1539 & -0.1570 & -0.1679 & 0.0775 & -0.2867 & 0.1781 & -0.0073 & 0.4135 \\
\hline & 0.0022 & 0.0018 & 0.0008 & 0.1246 & 0.0000 & 0.0004 & 0.8857 & 0.0000 \\
\hline
\end{tabular}

Notas: O p-valor está abaixo do coeficiente de correlação. As variáveis estatisticamente significantes estão em negrito. AD: JM, AD:KT e AD:DC são Accruals Discricionários estimados pelos modelos propostos por Dechow et al. (1995), Kothari et al. (2005) e Dechow et al. (2012). Qanali_t é o número de analistas que acompanharam a empresa i no tempo t; Erro_t-1_t-1 representa o erro da previsão da média dos analistas no ano anterior; Dotimer_t-1 é a interação de uma dummy que assume o valor 1 quando o erro foi menor do que zero (previsão otimista) com a magnitude do erro das previsões do ano anterior; Desvio_t diz respeito à variabilidade das previsões no ano corrente; ENDIV_t apresenta o endividamento da empresa no tempo $t$, medido pela razão entre as dívidas de curto e longo prazo e o ativo total; At_t representa o tamanho da empresa, aproximado pelo logaritmo natural do ativo total; $\mathbf{A D}$ representa os accruals discricionários.

É possível observar que a única proxy de accruals discricionários estatisticamente correlacionada $\left(0.1209^{* *}\right)$ com ERROPREV_t é a do novo modelo de Dechow et al. (2012). É importante também apontar a relação positiva e

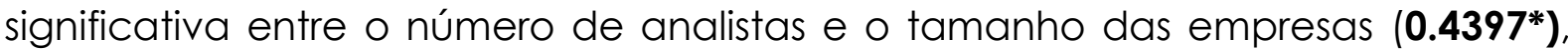
indicando que, em média, quanto maior a empresa, mais acompanhada pelos analistas ela é. As correlações entre as medidas de gerenciamento de resultados (ADs) apresentam coeficiente de correlação maior que 0.96 .

\subsection{Regressões Lineares}

A Tabela 3, abaixo, evidencia os resultados das regressões estimados pela abordagem dos Efeitos Fixos. Cabe ressaltar que os testes de abordagem (Testes de Chow, Testes de Breush-Pagan e Hausman) apontaram os efeitos fixos como a 
abordagem mais ajustada e apropriada para os dados. De forma comparativa ao modelo Jones Modificado (AD: JM), apresentam-se também os resultados estimados com os accruals Discricionários (AD: KT) de Kothari, Leone e Wasley, (2005), bem como os (AD: DC) calculados conforme Dechow et al. (2012).

Tabela 3: Regressões da hipótese 1. Variáveis dependentes são os accruals discricionários.

\begin{tabular}{|c|c|c|c|}
\hline \multicolumn{4}{|c|}{ 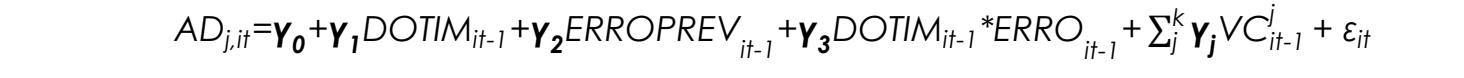 } \\
\hline \multirow{2}{*}{ Variáveis } & \multicolumn{3}{|c|}{ Var. Dep.: Accruals Discricionários (ADs) } \\
\hline & AD: JM & AD: KT & AD: DC \\
\hline \multirow{2}{*}{ Dotim_t-1 } & 0.0783 & 0.0840 & 0.0994 \\
\hline & $(0.133)$ & $(0.143)$ & $(0.179)$ \\
\hline \multirow{2}{*}{ Erroprev_t-1 } & 0.0106 & 0.0116 & 0.0131 \\
\hline & $(0.0963)$ & $(0.105)$ & $(0.126)$ \\
\hline \multirow{2}{*}{ Dotimerro_t-1 } & -1.911 & -2.090 & -2.378 \\
\hline & $(-0.0942)$ & $(-0.103)$ & $(-0.124)$ \\
\hline \multirow{2}{*}{ At_t-1 } & $0.0527^{*}$ & $0.0532^{*}$ & $0.0686 * * *$ \\
\hline & $(1.947)$ & $(1.965)$ & $(2.677)$ \\
\hline \multirow{2}{*}{ Qanalistas_t-1 } & -0.00488 & -0.00485 & -0.00506 \\
\hline & $(-1.379)$ & $(-1.372)$ & $(-1.510)$ \\
\hline \multirow{2}{*}{ Desviopadrao_t-1 } & 0.00480 & 0.00435 & 0.00887 \\
\hline & $(0.143)$ & $(0.130)$ & $(0.279)$ \\
\hline \multirow{2}{*}{ Endiv_t-1 } & -0.00906 & -0.00882 & $-0.0114^{*}$ \\
\hline & $(-1.259)$ & $(-1.226)$ & $(-1.670)$ \\
\hline \multirow{2}{*}{ MTB_t-1 } & -0.000579 & -0.000730 & 0.000721 \\
\hline & $(-0.0969)$ & $(-0.122)$ & $(0.127)$ \\
\hline \multirow{2}{*}{ Constant } & $-1.154^{*}$ & $-1.169 *$ & $-1.518^{* * *}$ \\
\hline & $(-1.935)$ & $(-1.960)$ & $(-2.690)$ \\
\hline Num. Obs. & 394 & 394 & 394 \\
\hline $\mathrm{R}^{2}$ & $2.30 \%$ & $2.30 \%$ & $3.80 \%$ \\
\hline Num. id & 80 & 80 & 80 \\
\hline$F$ & 0.913 & 0.919 & 1.503 \\
\hline
\end{tabular}

Notas: (i) Estatísticas t entre parênteses e significância estatística: ${ }^{* * *} p<0.01,{ }^{* *} p<0.05,{ }^{*} p<0.10$; (ii). As variáveis estatisticamente significantes estão em negrito. AD: JM, AD:KT e AD:DC são Accruals Discricionários estimados pelos modelos propostos por Dechow et al. (1995), Kothari et al. (2005) e Dechow et al. (2012). Qanali_t é o número de analistas que acompanharam a 
empresa i no tempo t; Erro_t-1_t-1 representa o erro da previsão da média dos analistas no ano anterior; Dotimer_t-1 é a interação de uma dummy que assume o valor 1 quando o erro foi menor do que zero (previsão otimista) com a magnitude do erro das previsões do ano anterior; Desvio_ł diz respeito à variabilidade das previsões no ano corrente; ENDIV_t apresenta o endividamento da empresa no tempo $t$, medido pela razão entre as dívidas de curto e longo prazo e o ativo total; At_t representa o tamanho da empresa, aproximado pelo logaritmo natural do ativo total; $A D$ representa os accruals discricionários.

Com base nos resultados da tabela 3, observa-se que nenhum dos modelos apresentou relação significativa esperada para as variáveis de interesse (Dotim_t1 e Dotim_t-1*Erro_t-1). No modelo de reversão dos accruals (última coluna), o poder explicativo foi de $3.80 \%$. Somente no modelo utilizando os (AD: DC) é que a variável Endividamento foi significativa e negativa a 10\%, conforme expectativa de maior monitoramento de credores, o que tende a desincentivar os gestores a gerenciar os resultados. Já o tamanho das empresas teve coeficiente positivo estatisticamente significante a $10 \%$ nos dois primeiros modelos $(0,0527$ e 0,0532$)$ e a $1 \%(0,0686)$ no terceiro modelo.

No intuito de verificar a hipótese 2, a tabela 4 apresenta os resultados dos coeficientes estimados também sob a abordagem dos Efeitos Fixos. O modelo também foi estimado utilizando a variáveis alternativas Accruals Discricionários (DECHOW et al., 2012; KOTHARI; LEONE; WASLEY, 2005).

Tabela 4: Regressão da hipótese 2. Variável dependente Erro de Previsão.

\begin{tabular}{|c|c|c|c|}
\hline ERROPREV $_{\text {it }}=\boldsymbol{Y}_{0}+\boldsymbol{Y}_{1} A D_{\text {it- }}$ & $+Y_{3} E R R C$ & $T I M_{i t-1}{ }^{*} E R F$ & $\sum_{j}^{k} \boldsymbol{Y}_{j} \vee C_{i t-1}^{j}$ \\
\hline Yruín' & & p.: ErroPre & \\
\hline vanioreis & AD: JM & AD: KT & $A D: D C$ \\
\hline$\overline{A D_{-} \dagger}$ & 7.092 & 7.052 & 7.037 \\
\hline & (1.509) & $(1.501)$ & $(1.443)$ \\
\hline Dotim_t-1 & 44.37 & 44.52 & 43.97 \\
\hline & $(0.776)$ & $(0.778)$ & (0.768) \\
\hline Erroprev_t-1 & 8.537 & 8.567 & 8.462 \\
\hline & (0.798) & $(0.801)$ & (0.790) \\
\hline Dotim_t-1 ${ }^{*}$ Erroprev_t-1 & $-1,619$ & $-1,625$ & $-1,606$ \\
\hline & $(-0.821)$ & $(-0.824)$ & $(-0.814)$ \\
\hline At_t-1 & 0.0719 & 0.0682 & 0.176 \\
\hline & $(0.0273)$ & $(0.0260)$ & $(0.0670)$ \\
\hline Qanalistas_t-1 & 0.198 & 0.198 & 0.188 \\
\hline & $(0.576)$ & $(0.577)$ & $(0.545)$ \\
\hline Desviopadrao_t-1 & 2.745 & 2.739 & 2.729 \\
\hline & $(0.836)$ & $(0.834)$ & (0.831) \\
\hline Endiv_t-1 & -0.248 & -0.252 & -0.256 \\
\hline & $(-0.354)$ & $(-0.360)$ & $(-0.366)$ \\
\hline MTB_t-1 & 0.486 & 0.488 & 0.472 \\
\hline
\end{tabular}


(0.839)

Constant

Num. Obs.:

$\mathrm{R}^{2}$

Num. id

F-estat
$(-0.234)$

394

$6.20 \%$

80

$2.227^{* *}$
(-0.233)

394

$6.20 \%$

80

$2.224^{* *}$
(0.814)

$-15.74$

$(-0.272)$

394

$6.10 \%$

80

$2.204^{* *}$

Notas: (i) Estatísticas t entre parênteses e significância estatística: ${ }^{* * *} p<0.01,{ }^{* *} p<0.05,{ }^{*} p<0.10$; (ii). As variáveis estatisticamente significantes estão em negrito. AD: JM, AD:KT e AD:DC são Accruals Discricionários estimados pelos modelos propostos por Dechow et al. (1995), Kothari et al. (2005) e Dechow et al. (2012). Qanali_t é o número de analistas que acompanharam a empresa i no tempo t; Erro_t-1_t-1 representa o erro da previsão da média dos analistas no ano anterior; Dotimer_t-1 é a interação de uma dummy que assume o valor 1 quando o erro foi menor do que zero (previsão otimista) com a magnitude do erro das previsões do ano anterior; Desvio_ł diz respeito à variabilidade das previsões no ano corrente; ENDIV_t apresenta o endividamento da empresa no tempo t, medido pela razão entre as dívidas de curto e longo prazo e o ativo total; At_t representa o tamanho da empresa, aproximado pelo logaritmo natural do ativo total; $A D$ representa os accruals discricionários.

Os resultados dos modelos estimados sob a abordagem dos efeitos fixos não permitem afirmar que a utilização dos accruals discricionários está estatisticamente relacionada à diminuição dos erros de previsão da média dos analistas, uma vez que em nenhum dos coeficientes das proxies utilizadas para ADs se mostrou significativo. Convém ressaltar que os modelos foram avaliados com diferentes cálculos de Accruals Totais (DECHOW; DICHEV, 2002; GIVOLY; HAYN, 2000), utilizados para a estimação dos Accruals Discricionários. Além disso, conforme apresentado na estatística descritiva, existem accruals discricionários negativos no p.25 e positivo em p.75 sugerindo que grupos de empresas manipulam para baixo e para cima os resultados, respectivamente.

Adicionalmente, foram testadas outras regressões (não reportadas) com estimadores alternativos (OLS com erros-padrão clusterizados na firma e no ano, OLS pooled com variáveis dummy de ano e setor e regressões quantílicas), porém os resultados mantiveram-se qualitativamente similares.

Uma possível explicação para a falta de relação estatística entre as previsões otimistas e o gerenciamento de resultado para atingir a expectativa do mercado no ano seguinte é a alta magnitude dos erros de previsão, mesmo após o tratamento dos outliers. Tal como no modelo 1, não foram encontrados resultados significativamente divergentes. É importante ressaltar que os resultados se mantiveram quando foram selecionados anos específicos ou excluídos os anos de 2008 e 2009.

Como análise de robustez, os modelos 1 e 2 foram estimados novamente apenas para as empresas que tiveram previsões otimistas, que representam 83,68\% da amostra de acordo com as estatísticas descritivas da variável Dotim_t1. Os resultados (não reportados) foram similares aos previamente apresentados. Em testes adicionais e não tabulados (disponíveis sob solicitação), foram incluídas variáveis explicativas em $t$, ao invés de $t-1$, mas os resultados observados foram qualitativamente semelhantes. 
Por meio dos diversos testes neste estudo, além dos elaborados por Mota et al. (2017), a questão da relação entre as previsões dos analistas de mercado e o gerenciamento de resultados ainda continua em aberto demandando estudos futuros.

\section{CONSIDERAÇÕES FINAIS}

O gerenciamento de resultados é uma prática amplamente difundida, praticada por diversas empresas por meio de maneiras diferentes. Este artigo, no entanto, abordou especificamente o gerenciamento dos accruals discricionários. Assim, o objetivo do estudo foi investigar a relação de incentivo entre as previsões otimistas dos analistas de mercado e a utilização desses accruals.

Buscou-se essa relação no intuito de verificar uma vertente da ideia lançada pelo estudo de Beaver, Lambert e Dale (1980) no que tange aos preços conduzirem os lucros, porém, pelo prisma das previsões dos analistas. Ainda, estudos anteriores demostraram evidências de gerenciamentos de resultados no Brasil, porém, não foi encontrado algum que tenha pesquisado a influência que as previsões otimistas do ano anterior podem provocar nas escolhas dos gestores sobre gerenciamentos de resultados para o atingimento de metas no período seguinte.

O primeiro modelo não permitiu confirmar a hipótese de que previsões otimistas, isto é, o não atingimento do consenso dos analistas, aproximado pela média das previsões, representa um incentivo à utilização de accruals discricionários no período subsequente. Também não se observou impacto da magnitude do erro otimista dos analistas em relação à utilização dos accruals discricionários.

No segundo modelo, o interesse foi verificar se tal gerenciamento diminuiria o erro das previsões no ano posterior, fazendo supor que as empresas estariam buscando atingir as expectativas por meio do gerenciamento, ou pelo menos tentando. Contudo, os achados não permitiram confirmar essa segunda hipótese.

Embora não tenha sido o foco deste estudo, sugere-se controlar a amostra com base na estrutura de governança corporativa no que tange à independência do conselho de administração, uma vez que há resultados significativos no campo da governança corporativa. A literatura concernente ao assunto mostra que participantes externos $e$ independentes atuam como monitores, impedindo ações de gerenciamento de resultados (HOLANDA; COELHO, 2016). Por fim, a relação entre gerenciamento de resultados e previsões dos analistas ainda demanda futuros estudos para consolidar o entendimento sobre o tema.

\section{REFERÊNCIAS}

ABARBANELL, J.; LEHAVY, R. Can Stock Recommendations Predict Earnings Management and Analysts' Earnings Forecast Errors? Journal of Accounting Research, v. 41, n. 1, p. 1-31, 1 mar. 2003.

\section{ALMEIDA, J. E. F. DE et al. EARNINGS MANAGEMENT NO BRASIL: GRUPOS}


ESTRATÉGICOS COMO NOVA VARIÁVEL EXPLANATÓRIA. Revista de Contabilidade e Organizações, v. 3, n. 6, p. 81-95, 24 set. 2009.

ALMEIDA, J. E. F. DE; DALMÁCIO, F. Z. The Effects of Corporate Governance and Product Market Competition on Analysts' Forecasts: Evidence from the Brazilian Capital Market. The International Journal of Accounting, v. 50, n. 3, p. 316-339, set. 2015.

ALMEIDA, J. E. F. DE; LOPES, A. B.; CORRAR, L. J. Gerenciamento de resultados para sustentar a expectativa do mercado de capitais: impactos no índice market-to-book. Advances in Scientific and Applied Accounting, v. 4, n. 1, p. 44$62,2011$.

ATHANASAKOU, V.; STRONG, N.; WALKER, M. Earnings management or forecast guidance to meet analyst expectations? Accounting and Business Research, $n$. 39, p. 3-35, 2009.

BARTOV, E.; GUL, F. A.; TSUI, J. S. L. Discretionary accruals models and audit qualifications. Journal of Accounting and Economics, n. 30, p. 421-452., 2001.

BARUA, A.; JOSEPH LEGORIA; MOFFITTI, J. S. Accruals Management to Achieve Earnings Benchmarks: A Comparison of Pre-managed Profit and Loss Firms. Journal of Business Finance \& Accounting, v. 33, n. 5 e 6, p. 653-670, jul. 2006.

BASU, S.; HWANG, L.; JAN, C. International Variation in Accounting Measurement Rules and Analysts' Earnings Forecast Errors. Journal of Business Finance \& Accounting, v. 25, n. 9-10, p. 1207-1247, 1998.

BEAVER, W. H. Perspectives on Recent Capital Market Research. The Accounting Review, v. 77, n. 2, p. 453-474, 2002.

BEAVER, WILLIAM; LAMBERT, RICHARD; DALE, M. The information content of the price. Journal of Accounting and Economics, v. 2, p. 3-28, 1980.

COMPRIX, J.; MULLER, K.; STANFORD, M. Economic Consequences of Mandatory Adoption of IAS in the European Union. Working paper, Pennsylvania State University, 2003.

CRAGG, J. G.; MALKIEL, B. G. The Consensus and Accuracy of Some Predictions of the Growth of Corporate Earnings. The Journal of Finance, v. 23, n. 1, p. 67-84, mar. 1968.

DALMÁCIO, F. Z. et al. An analysis of the relationship between corporate governance and accuracy of the analysts forecasts of the Brazilian market. RAM. Revista de Administração Mackenzie, v. 14, n. 5, p. 104-139, out. 2013.

DECHOW, P. M. et al. Detecting Earnings Management: A New Approach. Journal of Accounting Research, v. 50, n. 2, p. 275-334, 1 maio 2012.

DECHOW, P. M.; DICHEV, I. D. The quality of accruals and earnings: The role of accrual estimation errors. The accounting review, v. 77, n. s-1, P. 35-59, 2002. 
DECHOW, P.M.; SLOAN, R. G.; SWEENEY, A. P. Detecting Earnings Management. The Accounting Review, v. 70, p. 193-225, 1995.

DEGEORGE, F.; PATEL, J.; ZECKHAUSER, R. Earnings Management to Exceed Thresholds. The Journal of Business, v. 72, n. 1, p. 1-33, 1999.

ERFURTH, A. E.; BEZERRA, F. A. GERENCIAMENTO DE RESULTADOS NOS DIFERENTES NÍVEIS DE GOVERNANÇA CORPORATIVA. Revista Base (Administração e Contabilidade) da UNISINOS, v. 10, n. 1, 2013.

FÁVERO, L. P. et al. Análise de dados. Modelagem multivariada para tomada de decisões. 7. ed. São Paulo: Elsevier, 2009.

GIVOLY, D.; HAYN, C. The changing time-series properties of earnings, cash flows and accruals: Has financial reporting become more conservative? Journal of Accounting and Economics, v. 29, n. 3, p. 287-320, 2000.

GIVOLY, D.; HAYN, C.; YODER, T. R. Do Analysts Account for Earnings Management? Rochester, NY: Social Science Research Network, 1 nov. 2011. Disponível em: <http://papers.ssrn.com/abstract=1260032>. Acesso em: 12 ago. 2014.

HOLANDA, A. P.; COELHO, A. C. GERENCIAMENTO DE RESULTADOS E ESTRUTURA DE PROPRIEDADE: EVIDÊNCIAS NO BRASIL. REVISTA REUNIR, v. 6, n. 2, p. 17-35, 1 set. 2016.

HOLANDA, A. P.; COELHO, A. C. D. Dividends and clientele effect: evidence in the Brazilian market. Revista de Administração de Empresas, v. 52, n. 4, p. 448463, ago. 2012.

JENSEN, M. C.; MECKLING, W. H. Theory of the firm: Managerial behavior, agency costs and ownership structure. Journal of Financial Economics, v. 3, n. 4, p. 305360, out. 1976.

KEUNG, E.; LIN, Z.-X.; SHIH, M. Does the Stock Market See a Zero or Small Positive Earnings Surprise as a Red Flag? Journal of Accounting Research, v. 48, n. 1, p. 91-121, 1 mar. 2010.

KOTHARI, S. P.; LEONE, A. J.; WASLEY, C. E. Performance matched discretionary accrual measures. Journal of Accounting and Economics, v. 39, n. 1, p. 163-197, fev. 2005.

LEE, J. Earnings management to just meet analysts' forecasts. (Working paper) Kellogg Graduate School of Management., 2007.

LIMA, A. S. DE et al. Life Cycle Stages and Earnings Quality in Brazil. Revista de Administração Contemporânea, v. 19, n. 3, p. 398-418, jun. 2015.

MARTINEZ, A. L. Earnings management. Empirical study of the Brazilian public companies. text-[s.I.] Universidade de São Paulo, 2001. 
MARTINEZ, A. L. Análise da Surpresa dos Analistas Ao Anúncio dos Resultados Contábeis: Evidências Empíricas Para As Companhias Abertas Brasileiras. 4 Congresso USP de Controladoria e Contabilidade, n. 4, 2004.

MARTINEZ, A. L. Otimismo e Viés de Seleção dos Analistas. Brazilian Business Review, v. 4, n. 2, p. 104-118, ago. 2007.

MARTINEZ, A. L. Determinantes da Acurácia das Previsões dos Analistas do Mercado de Capitais. Contabilidade, Gestão e Governança, v. 10, n. 2, 3 dez. 2009.

MARTINEZ, ANTONIO LOPO. Cobertura de Analistas, Erros de Previsão e Earnings Management no Brasil. XXXIII Encontro da ANPAD, n. 13, 2009.

MARTINS, V. G.; PAULO, E.; MONTE, P. A. DO. O GERENCIAMENTO DE RESULTADOS CONTÁBEIS EXERCE INFLUÊNCIA NA ACURÁCIA DA PREVISÃO DE ANALISTAS NO BRASIL? Revista Universo Contábil, v. 12, n. 3, p. 73-90, 27 set. 2016.

MATSUMOTO, D. A. Management's Incentives to Avoid Negative Earnings Surprises. The Accounting Review, v. 77, n. 3, p. 483-514, 1 jul. 2002.

MOTA, R. H. G. et al. PREVISÃO DE LUCRO E GERENCIAMENTO DE RESULTADOS: EVIDÊNCIAS EMPÍRICAS NO MERCADO ACIONÁRIO BRASILEIRO. Revista Universo Contábil, v. 13, n. 1, p. 06-26, 25 abr. 2017.

OLIVEIRA, A. S. DE; GIRÃO, L. F. DE A. P. Acurácia na previsão de lucros e os estágios do ciclo de vida: evidências no mercado brasileiro de capitais. $\mathrm{XI}$ Congresso Anpcont. Anais...Ribeirão Preto - SP: 2016Disponível em: <http://congressos.anpcont.org.br/x/anais/files/2016-05/mfc319.pdf>. Acesso em: 29 out. 2017

PLUMMER, E.; MEST, D. P. Evidence on the Management of Earnings Components. Journal of Accounting, Auditing \& Finance, v. 16, n. 4, p. 301-323, 1 out. 2001.

RONEN, J.; YAARI, V. Earnings Management. Emerging Insights in Theory, Practice, and Research. [s.l.] Springer Series in Accounting Scholarship, 2008. v. 3

SAITO, R.; BENETTI, S. J. S. V. E C.; VILLALOBOS, SONIA JULIA SULZBECK. Qualidade das projeções dos analistas sell-side: evidência empírica do mercado brasileiro. Revista de Administra\&ccdeil;ão da Universidade de São Paulo, v. 43, n. 4, 2008.

SILVA, V. C. D. A adoção das ifrs impacta no gerenciamento de resultados? Evidências do mercado de capitais brasileiro. Dissertação (Mestrado em Ciências Contábeis)_Vitória/ES: Universidade Federal do Espírito Santo, 2015. 